\title{
Vowel Dispersion in Truku
}

\author{
Wen-yu Chiang and Fang-mei Chiang \\ National Taiwan University
}

\section{Introduction}

This study investigates the dispersion of vowel space in Truku, an endangered Austronesian language in Taiwan. Adaptive Dispersion (Liljencrants and Lindblom, 1972; Lindblom, 1986, 1990) proposes that the distinctive sounds of a language tend to be positioned in phonetic space in a way that maximizes perceptual contrast. For example, languages with large vowel inventories tend to expand the overall acoustic vowel space. Adaptive Dispersion predicts that the distance between the point vowels will increase with the size of a language's vowel inventory. Thus, the available acoustic vowel space is utilized in a way that maintains maximal auditory contrast.

Flemming $(1995,1996,2001)$ introduced another version of Dispersion Theory, which incorporates the mechanisms of dispersion into current phonological theories of constraints on development of phonological inventories. Expressed in terms of Optimality Theory, this version of Dispersion theory claims that constraints favoring less perceptually confusable contrasts are ranked higher than constraints that would favor more confusable contrasts. Thus, the markedness of a sound would depend on the sounds that it contrasts with in a particular inventory. Flemming proposes three central constraints: i) Maximize the distinctiveness of contrasts; ii) Minimize articulatory effort, and iii) Maximize the number of contrasts. The particular requirements of each language's inventory determine the order in which these three constraints are ranked, which results in divergent language-specific vowel systems.

The current study provides unique data in Truku, which is particularly suited to testing dispersion theory, because they reveal conflicting opinions as to the number of vowels in the Truku inventory. In the scant literature currently available, Truku is inconsistently claimed to contain three vowels $(/ \mathrm{i} /, / \mathrm{u} /$ and $/ \mathrm{a} /)$, four vowels $(/ \mathrm{i} /, / \mathrm{u} /, / \mathrm{o} /$ and $/ \mathrm{a} /)$ or five vowels $(/ \mathrm{i} /, \mathrm{e} /, / \mathrm{u} /, / \mathrm{o} /$ and $/ \mathrm{a} /)$, not including the schwa. Moreover, many native speakers of Truku also differ with respect to their perception of the number of vowels in its inventory. The current study investigates two research questions: 1) whether dispersion theory can account for variations in the reported number of Truku vowels and 2) how syllabic stress may interact with the vowels' distribution and contrastiveness.

\section{Background}

In terms of linguistic typology, Truku is described as a descendant of proto-Atayal. Due to its long alienation from Atayal, Truku diverges considerably from its prototype. Truku had no writing system, which is common to all Formosan aboriginal languages, so evidence of diachronic change cannot be determined from written records. The Atayal tribe can be divided into two subtypes: Atayal and Seediq (Li et al, (1963) and Hung (1993)). The latter, further divided into Eastern and Western Seediq, are dispersed over Taiwan's Hualien and Nantou Counties. The languages spoken 
by the Seediq can be grouped into three dialects: Teuda, Tkdaya and Truku, which belonged to the Eastern Seediq. The Eastern Seediq population of Hualien is 24,183 , which represents around $84 \%$ of the entire Seediq population (Huang, 1990).

In recent years, debate has arisen as to the composition of the Truku vowel inventory. The Truku vowel system, in the divergent opinions of researchers, consists of three, four or five vowels. ${ }^{1}$ Hu (2003) claims that the Truku inventory consists of the three vowels $/ \mathrm{i} /, / \mathrm{u} /$ and $/ \mathrm{a} /$. The ALCD (Center for Aboriginal Languages Cultures Education, 2001) classifies Truku as having the four vowels $/ \mathrm{i} /, / \mathrm{u} /, / \mathrm{o} /$ and $/ \mathrm{a} / . \quad \mathrm{Li}$ (1992) claimed that all three dialects of Seediq have $/ \mathrm{i} /, / \mathrm{u} /, / \mathrm{o} /$ and $/ \mathrm{a} /$, while the occurrence of $/ \mathrm{e} / \mathrm{or} / \mathrm{o} / \mathrm{depends}$ on regional variation. ${ }^{2}$ Yang (1976) and Chang (2000) consider all dialects of Seediq to have a five-vowel system, consisting of $/ \mathrm{i} /, \mathrm{e} /, / \mathrm{u} /, / \mathrm{o} /$ and $/ \mathrm{a} /$.

The writing system of Truku language is currently undergoing the process of construction through the discussion of its native speakers. Disagreement concerning the number of phoneme emerges as an unresolved problem. The current study thus aims to investigate the relationship between the number of vowel inventory and the distribution of vowel space.

\section{Method}

\section{Materials}

A list of seventy-seven disyllabic words containing the vowels [i], [I]/[E], [u] and $[\mathrm{U}] /[\mathrm{O}]$ were selected from our field recordings. Forty-four were presented in stressed conditions and thirty-three in unstressed conditions. ${ }^{3}$ The wordlist, including vowel occurrence tabulations, are given in Table 1 . The words were selected specifically to include as many contexts as possible, in order to include the possibility of coarticulation effects. Gaps in this list result from either the lack of lexical item to fulfill that condition, or the absence of an acceptable token of such an item in our field recordings ${ }^{4}$. Forty disyllabic words containing the vowels [a] and [ə] were added to test the integrity of the vowel chart. The column of unstressed [ə] in Table 1 is empty; our consultations with informants yielded no example in which [ə] can appear as the second syllable of a disyllabic word.

We observed that [i], [u] and [a] were the distinctive vowels upon which all speakers agreed, unlike [e] and [o]. The controversial nature of [e] and [o] was marked by the alternating use of capital " $\mathrm{I}$ " and " $\mathrm{E}$ " for the former, and " $\mathrm{U}$ " and " $\mathrm{O}$ " for the latter, the capital letters representing the underlying form as recognized by different groups of speakers.

Table 1a. Items representing stressed vowels in Truku

\begin{tabular}{|c|c|c|c|c|}
\hline $\begin{array}{l}\text { Preceding } \\
\text { Consonants }\end{array}$ & [i] & {$[\mathbf{I}] /[\mathbf{E}]$} & [u] & {$[\mathbf{U}] /[\mathbf{O}]$} \\
\hline $\mathrm{p}$ & pila 'money' & pedang '[name]' & pungu 'knee' & poli '[name]' \\
\hline b & birat 'ear' & & $\begin{array}{l}\text { bunga'sweet } \\
\text { potato' }\end{array}$ & boyak 'boar' \\
\hline $\mathrm{m}$ & mirit 'goat' & meyaw 'awake' & muhing 'nose' & moda 'to pass' \\
\hline $\mathrm{t}$ & tcimu 'salt' & temu '[name]' & tunux 'head' & \\
\hline d & & & & dohong 'mortar' \\
\hline
\end{tabular}




\begin{tabular}{|c|c|c|c|c|}
\hline $\mathrm{n}$ & \multicolumn{2}{|l|}{ nisu 'your' } & nunuh 'a fig' & noxay 'because' \\
\hline $\mathbf{k}$ & \multicolumn{2}{|l|}{ kingal 'one' } & kumay 'bear' & \\
\hline $\mathrm{g}$ & \multirow{2}{*}{\multicolumn{2}{|c|}{$\begin{array}{l}\text { gimi 'to find' } \\
\text { ngiraw 'mushroom' }\end{array}$}} & gupun 'teeth' & gomuk 'a lid' \\
\hline ng & & & ngudus 'beard' & \\
\hline q & & & $q u y u$ 'snake' & qomi 'needle' \\
\hline $\mathrm{r}$ & risaw 'boy' & rema 'five' & & rodox 'chicken' \\
\hline 1 & lingis 'to cry' & & lungaw 'bottle' & lomun 'liver' \\
\hline $\mathrm{y}$ & & yeku '[name]' & yumaw'[name]' & yona '[name]' \\
\hline w & wihe 'spoon' & & & \\
\hline $\mathrm{h}$ & hiya 'he' & heni 'here' & & holing 'dog' \\
\hline $\mathbf{s}$ & sida 'branch' & & sudu 'grass' & soki 'knife' \\
\hline $\mathrm{z}$ & zima 'banboo' & & & \\
\hline Total & 14 & 6 & 12 & 12 \\
\hline
\end{tabular}

Table 1b. Items representing unstressed vowels in Truku

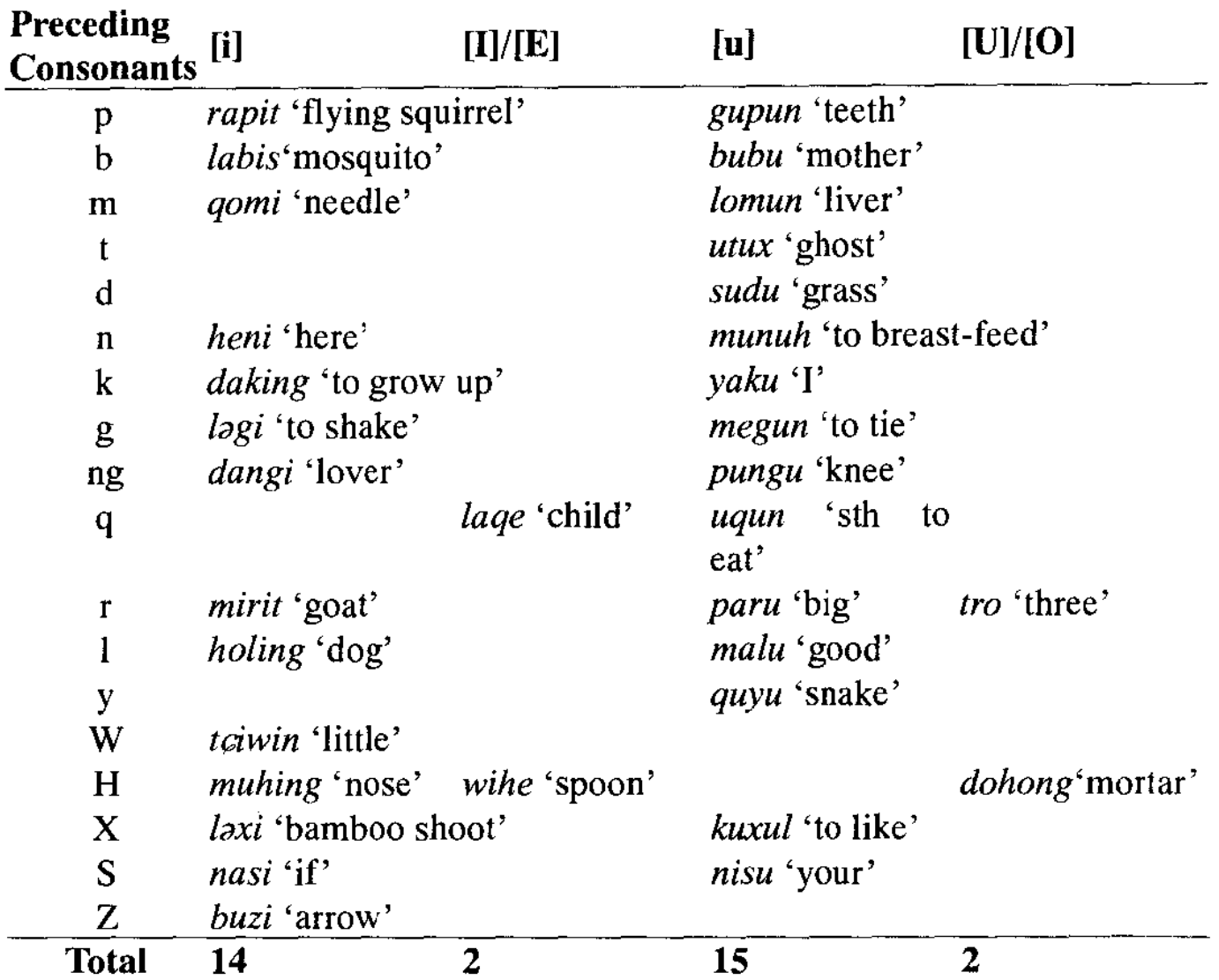

Table 1c. Items containing the vowels [a] and [o] in Truku

\begin{tabular}{|c|c|c|c|}
\hline Preceding & & tressed & Unstressed \\
\hline Consonants [ & [a] & [ə] & [a] \\
\hline $\mathrm{p}$ & paru 'big' & & sapah 'house' \\
\hline b & baraw 'upper' & & yabas 'guava' \\
\hline $\mathrm{m}$ & maxal 'ten' & mokan 'to eat' & tama 'father' \\
\hline $\mathrm{t}$ & tama 'father' & təro 'three' & watan '[name]' \\
\hline d & daha 'two' & domux' grains of' & idas 'moon' \\
\hline $\mathbf{n}$ & naku 'my' & & kana 'all' \\
\hline $\mathrm{k}$ & kari 'language' & kəla 'to understan & \\
\hline
\end{tabular}




\begin{tabular}{|c|c|c|c|}
\hline $\begin{array}{c}\mathrm{g} \\
\mathrm{ng}\end{array}$ & $\begin{array}{l}\text { gaga 'that' } \\
\text { ngali 'to take' }\end{array}$ & gohak 'seed' & $\begin{array}{l}\text { baga 'hand' } \\
\text { bunga 'sweet potato' }\end{array}$ \\
\hline $\mathrm{q}$ & qalux 'balck' & $q \partial p i^{\prime}$ to squeeze' & niqan 'to own' \\
\hline $\mathrm{r}$ & rawa 'bascket' & & \\
\hline 1 & laqe 'child' & loxi 'bamboo shoot' & alang 'village' \\
\hline $\mathrm{y}$ & yamu 'you (pl)' & & sayang 'now; today' \\
\hline w & wadu 'honey' & & rawa 'bascket' \\
\hline $\mathbf{h}$ & $\begin{array}{l}\text { habuk 'weast } \\
\text { belt' }\end{array}$ & həngak 'air' & daha 'two' \\
\hline $\mathrm{x}$ & & & maxal 'ten' \\
\hline $\mathbf{S}$ & sari 'taro' & sopac 'four' & mosa 'this way' \\
\hline otal & 16 & 9 & 5 \\
\hline
\end{tabular}

\section{Speakers}

Three male and three female native speakers of Truku participated in this experiment. The participants were divided into "5-vowel", "3-vowel" and "4-vowel" groups, according to the number of vowels they perceived to be in their language; each group included one male and one female speaker. The 5 -vowel group perceived the vowels [i], [e], [a], [u] and [o] in Truku, while the 3 -vowel group perceived [i], [u] and [a]. The speakers in the 4-vowel group perceived [i], [u], [o] and [a]. Thus, the vowel inventory for the 5-vowel group was: [i], [E], [a], [u] and [O]; for the 3-vowel group: [i], [I], [a], [u] and [U]; and for uncertain group, [i], [I], [u] and [O].

All of the speakers lived in Hualien County, where they spoke Truku on a daily basis before coming to Taipei at the age of twenty or above.

\section{Recordings}

Recording sessions consisted of two parts. The first familiarized the informants with the items on the randomized list of words; the second directed the informants to read each word once in Truku after hearing the corresponding Mandarin gloss. Some items contained two experimental vowels; these appeared twice in the wordlist, but never adjacently. The recordings were conducted in quiet rooms using a Sony TCM 5000-EV portable tape recorder and an electric condenser microphone located approximately 20 centimeters away from the mouth.

\section{Measurements and Analysis}

The signals were later transferred to the Kay Elemetrics Computerized Speech Lab (CSL) in National Taiwan University's Phonetics Laboratory, using a $10-\mathrm{kHz}$ sampling rate. Each word was displayed on a wideband spectrogram with a formant history, using a $300 \mathrm{kHz}$ bandwidth cutoff. Using both visual and auditory cues, we removed the preceding and following consonantal transitions around the experimental vowel, so that only the steady state of each vowel remained. Then, five points within this stable range were extracted at equal distances: $0 \%, 25 \%, 50 \%, 75 \%$ and $100 \%$ of the steady-state duration. F1 and $\mathrm{F} 2$ values were obtained for each of these points by means of an LPC analysis. However, since the vowels $[\mathrm{e}]$ and [o] are often diphthongized, only the portion before their offglide was extracted. If formant values were unavailable for any of the default five points $(0 \%, 25 \%, 50 \%, 75 \%$ and $100 \%)$, 
the value of an adjacent point was adopted, which was determined by examination of the LPC formant history.

\section{Result}

The following sections will present vowel distribution across groups in terms of the factors vowel identity and gender, as produced in the stressed condition. First, Figures la to 1f show the vowel distribution of each speaker in the stressed condition. F1/F2 plots reveal that except for the 5-vowel female speaker (Fig. 1a), who has clear distinctions for all the vowels, the other speakers show overlap among vowels. This is especially apparent in Figure 1b; for the 3-vowel female speaker, the controversial and non-controversial vowels occupy almost the same space. Furthermore, the male speakers' overall vowel space appears to be articulated further back than the female speakers'.

a. 5V-F's vowel distribution

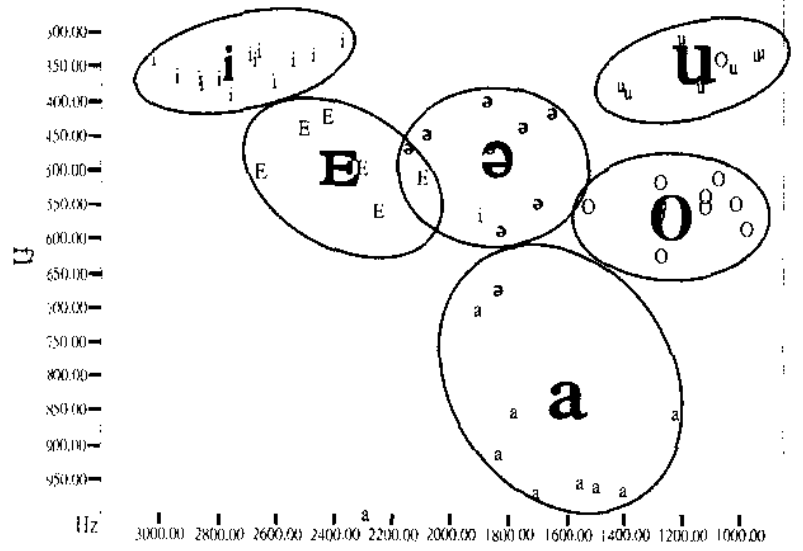

f2

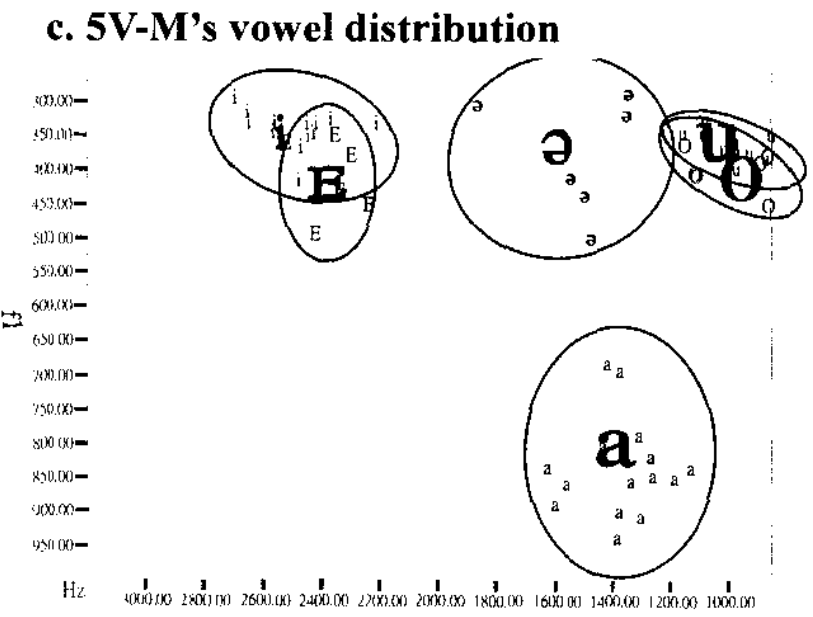

f2

\section{b. 3V-F's vowel distribution}

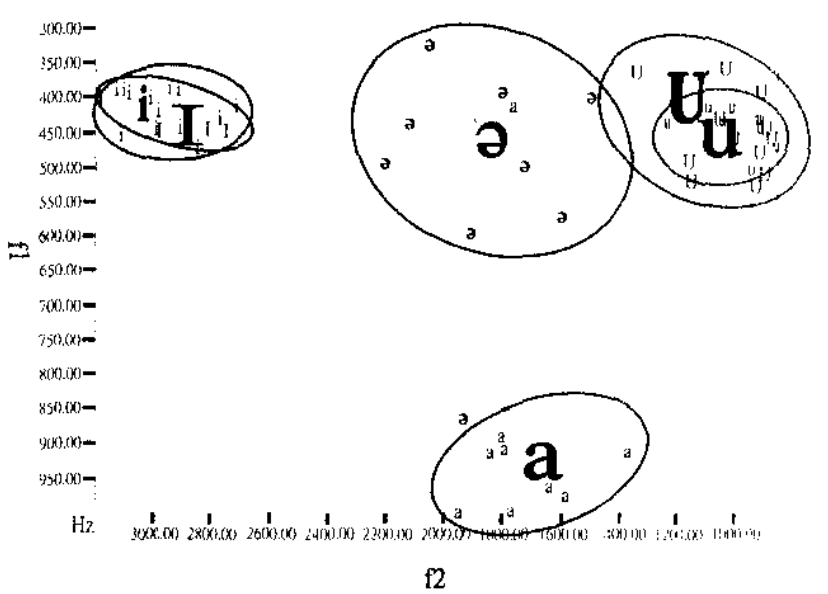

\section{d. 3V-M's vowel distribution}

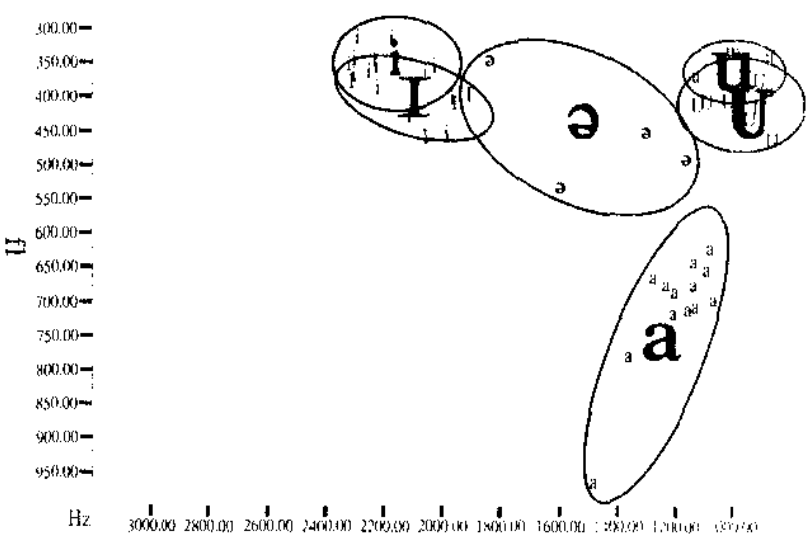

f2 


\section{e. 4V-F's vowel distribution}

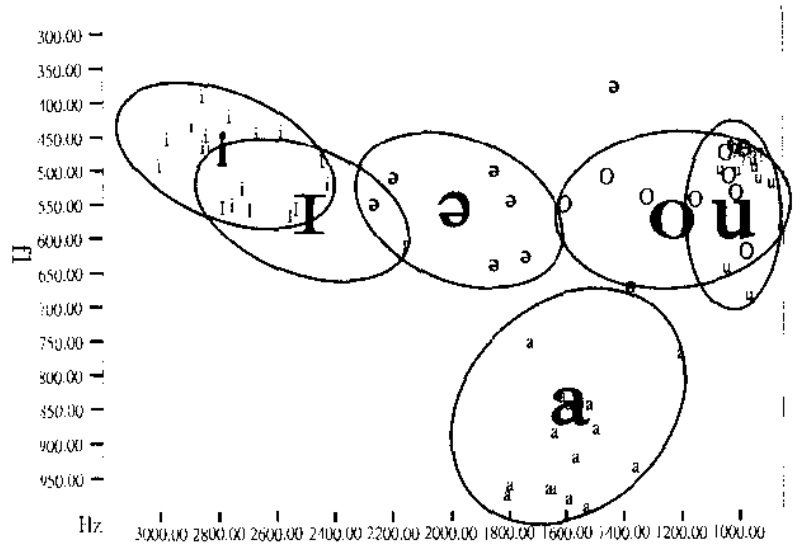

f2
1. f. $4 \mathrm{~V}-\mathrm{M}$ 's vowel distribution

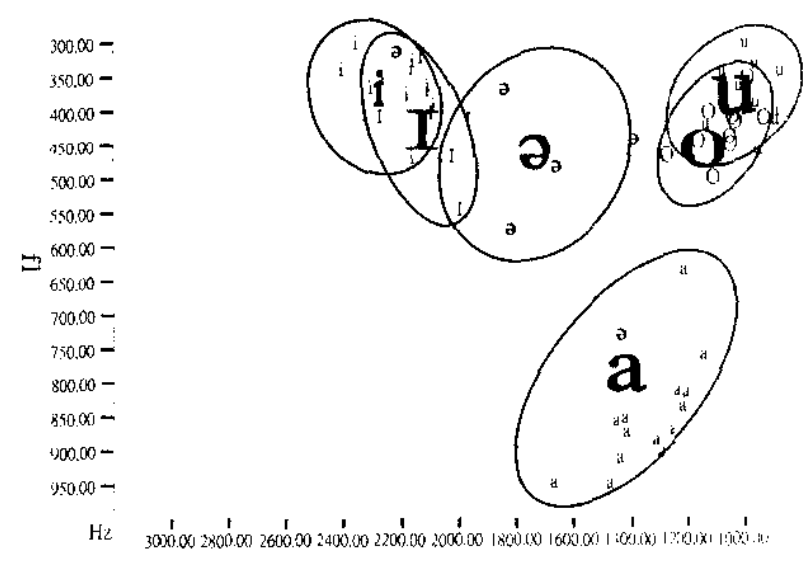

f2

Figure 1. The six speakers' individual vowel distributions in the stressed condition

Front vowels: [i] vs. [I/E]

Vowel and Gender factors.

The data were analyzed using a MANOVA with factors Vowel and Gender. With the F1 and F2 values as the dependent variable, there were significant effects for both factors. (For [i]-[I/E] difference: $\mathrm{F}(1,1051)=146.2, p<.01$; For female/male difference: $\mathrm{F}(1,1051)=653.2, p<.01)$. That is to say, the non-controversial [i] differ from the controversial [1/E] with respect to both $\mathrm{F} 1$ and F2 values despite the individual differences in perception. On the other hand, the factor of Gender is also significant for front vowels. The females' articulation of front vowels is lower and more fronted than the males'.

Within-subject variation.

We will now turn to the aspect of within-subject variation in comparing [i] and [I/E]. Table 2 provides the formant values and the ANOVA results for the comparison of the two front vowels [i] and [I/E].

Table 2. Formant values (Mean/SD) of the front vowels

\begin{tabular}{lllll}
\hline Mean (SD): $\mathbf{H z}$ & {$[\mathrm{i}]$} & {$[\mathbf{I} / \mathbf{E}]$} & \multicolumn{1}{c}{ ANOVA } \\
\hline \multirow{2}{*}{ 5V-Female } & $\mathrm{F} 1$ & $364(61)$ & $486(51)$ & $\mathrm{F}(1,98)=61.53, p<.001$ \\
& $\mathrm{~F} 2$ & $2649(280)$ & $2371(196)$ & $\mathrm{F}(1,98)=22.04, p<.001$ \\
\hline \multirow{2}{*}{ 4V-Female } & $\mathrm{F} 1$ & $464(44)$ & $556(41)$ & $\mathrm{F}(1,98)=66.11, p<.001$ \\
& $\mathrm{~F} 2$ & $2784(153)$ & $2529(217)$ & $\mathrm{F}(1,98)=26.86, p<.001$ \\
\hline \multirow{2}{*}{ 3V-Female } & $\mathrm{F} 1$ & $416(29)$ & $433(22)$ & $\mathrm{F}(1,88)=3.32, p=.072$ \\
& $\mathrm{~F} 2$ & $2951(129)$ & $2928(194)$ & $\mathrm{F}(1,88)=.314, p=.577$ \\
\hline \multirow{2}{*}{ 5V-Male } & $\mathrm{F} 1$ & $337(27)$ & $408(57)$ & $\mathrm{F}(1,98)=51.81, p<.001$ \\
& $\mathrm{~F} 2$ & $2490(127)$ & $2360(99)$ & $\mathrm{F}(1,98)=19.69, p<.001$ \\
\hline \multirow{2}{*}{ 4V-Male } & $\mathrm{F} 1$ & $366(41)$ & $425(75)$ & $\mathrm{F}(1,93)=23.99, p<.001$ \\
& $\mathrm{~F} 2$ & $2220(104)$ & $2076(113)$ & $\mathrm{F}(1,93)=24.12, p<.001$ \\
\hline \multirow{2}{*}{ 3V-Male } & $\mathrm{F} 1$ & $359(35)$ & $412(28)$ & $\mathrm{F}(1,98)=40.43, p<.001$ \\
& $\mathrm{~F} 2$ & $2171(123)$ & $2069(140)$ & $\mathrm{F}(1,98)=9.893, p<.005$ \\
\hline
\end{tabular}


According to ANOVA, most speakers' controversial [I/E] was significantly different from their non-controversial [i] in both $\mathrm{F} 1$ and $\mathrm{F} 2$ values; the only exception was 3-vowel female speaker, who made nearly no difference between the two vowels.

Back vowels: [u] vs. [U/O]

Vowel and Gender factors.

Likewise, there were significant effects for both factors Vowel and Gender on $\mathrm{F} 1$ and $\mathrm{F} 2$ values (For $[\mathrm{u}]-[\mathrm{U} / \mathrm{O}]$ difference: $\mathrm{F}(1,1216)=176.3, p<.01$; For female/male difference: $\mathrm{F}(1,1216)=97.5, p<.01)$. Therefore, the non-controversial $[\mathrm{u}]$ differ from the controversial [U/O] with respect to $\mathrm{F} 1$ and $\mathrm{F} 2$ values. This indicates that $[\mathrm{U} / \mathrm{O}]$ is lower and more fronted than the $[\mathrm{u}]$. As for the factor of Gender, the females' articulation of back vowels is lower and more fronted than the males'.

Within-subject variation.

As far as the within-subject variation is concerned, Table 3 provides the formant values and the ANOVA results for the comparison of the two back vowels $[\mathrm{u}]$ and $[\mathrm{U} / \mathrm{O}]$.

Table 3. Formant values (Mean/SD) of the back vowels

\begin{tabular}{lllll}
\hline Mean (SD): & Hz & {$[\mathbf{u}]$} & {$[\mathrm{U} / \mathrm{O}]$} & \multicolumn{1}{c}{ ANOVA } \\
\multirow{2}{*}{ 5V-Female } & $\mathrm{F} 1$ & $366(44)$ & $535(69)$ & $\mathrm{F}(1,118)=469.82, p<.001$ \\
& $\mathrm{~F} 2$ & $992(240)$ & $1190(154)$ & $\mathrm{F}(1,118)=24.87, p<.001$ \\
\hline \multirow{2}{*}{ 4V-Female } & $\mathrm{F} 1$ & $512(76)$ & $527(45)$ & $\mathrm{F}(1,118)=1.40, p=.239$ \\
& $\mathrm{~F} 2$ & $956(75)$ & $1065(217)$ & $\mathrm{F}(1,118)=5.57, p<.05$ \\
\hline \multirow{2}{*}{ 3V-Female } & $\mathrm{F} 1$ & $434(28)$ & $452(58)$ & $\mathrm{F}(1,118)=3.109, p=.08$ \\
& $\mathrm{~F} 2$ & $940(133)$ & $1012(144)$ & $\mathrm{F}(1,118)=6.275, p<.05$ \\
\hline \multirow{2}{*}{ 5V-Male } & $\mathrm{F} 1$ & $364(24)$ & $377(41)$ & $\mathrm{F}(1,118)=3.261, p=.073$ \\
& $\mathrm{~F} 2$ & $912(140)$ & $1077(444)$ & $\mathrm{F}(1,118)=7.727, p<.01$ \\
\hline \multirow{2}{*}{ 4V-Male } & $\mathrm{F} 1$ & $377(44)$ & $433(40)$ & $\mathrm{F}(1,113)=41.294, p<.001$ \\
& $\mathrm{~F} 2$ & $944(101)$ & $993(102)$ & $\mathrm{F}(1,113)=5.446, p<.05$ \\
\hline \multirow{2}{*}{ 3V-Male } & $\mathrm{F} 1$ & $368(38)$ & $409(30)$ & $\mathrm{F}(1,118)=32.365, p<.001$ \\
& $\mathrm{~F} 2$ & $889(113)$ & $900(127)$ & $\mathrm{F}(1,118)=.123, p=.727$ \\
\hline
\end{tabular}

In the back vowels' case, most speakers' controversial [U/O] and non-controversial $[\mathrm{u}]$ were significantly different mainly in $\mathrm{F} 2$ values, but not consistently in $F 1$ values.

The results showed that despite the individual difference in perception, the controversial vowels $([\mathrm{I} / \mathrm{E}]$ and $[\mathrm{U} / \mathrm{O}]$ ) were different from their non-controversial vowels ([i] and [u]). Moreover, the factor Gender also contributed to the variations among front and back vowels.

\section{Stress factor}

Figure 2 represents the speakers' vowel distribution in the unstressed condition. Although fewer tokens representing the controversial groups $[\mathrm{I} / \mathrm{E}]$ and $[\mathrm{U} / \mathrm{O}]$ were available, Figure 3 shows that some speakers separate the two pairs of vowels more clearly than others. The 5-vowel female, 4-vowel female and 4-vowel male made clearer distinctions than did the 5-vowel male, 3-vowel 
male and the 3 -vowel female. ${ }^{5}$ In addition, the male speakers have a more back and contracted vowel space than the female speakers do, which was also found in the stressed condition. ${ }^{6}$

Results of MANOVA showed that for the [i]-[I/E] pair, stress does not have a significant effect on the formant values produced, nor did stress interact with the factors Gender and Vowel Identity. In contrast, the effect of stress on formant production was found to be significant for the $[\mathrm{u}]-[\mathrm{U} / \mathrm{O}]$ pair $(\mathrm{F}(1$, $1216)=69.5, p<.01)$. We also found significant interaction of Stress with Gender and Vowel Identity for the [u]-[U/O] pair [Gender*Stress: $F(1,1216)=$ $4.8, p<.01$; Vowel Identity*Stress: $\mathrm{F}(1,1216)=39.8, p<.01]$. In sum, the formant values of the $[\mathrm{u}]-[\mathrm{U} / \mathrm{O}]$ pair were found to be influenced by stress, while those of $[\mathrm{i}]-[\mathrm{I} / \mathrm{E}]$ pair were not.

\section{a. 5V-F's vowel distribution}

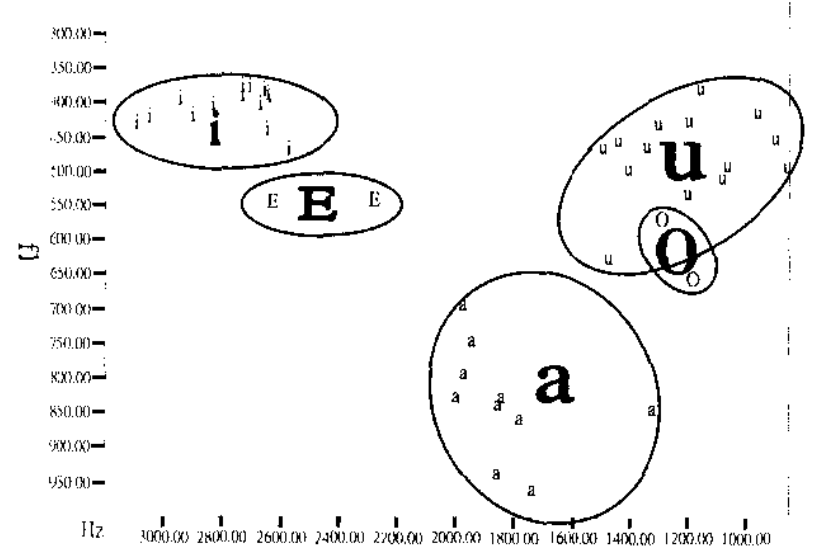

f2

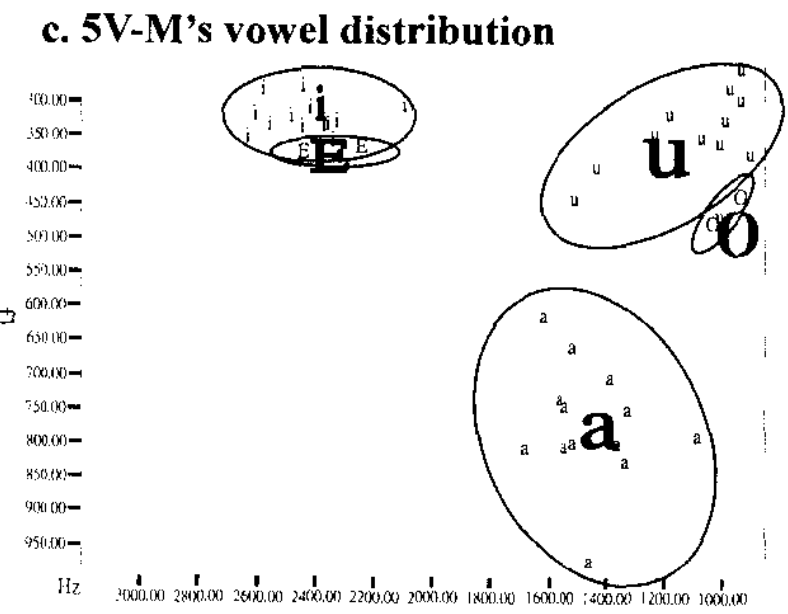

f2

\section{b. 3V-F's vowel distribution}

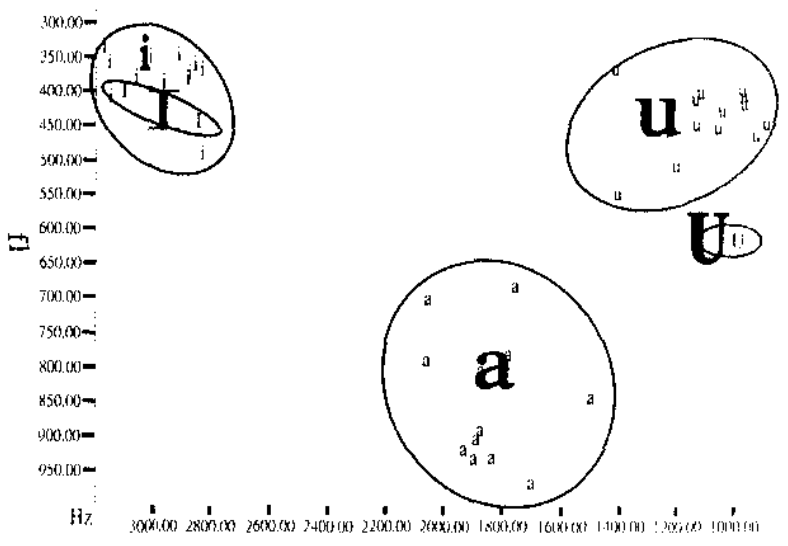

f2

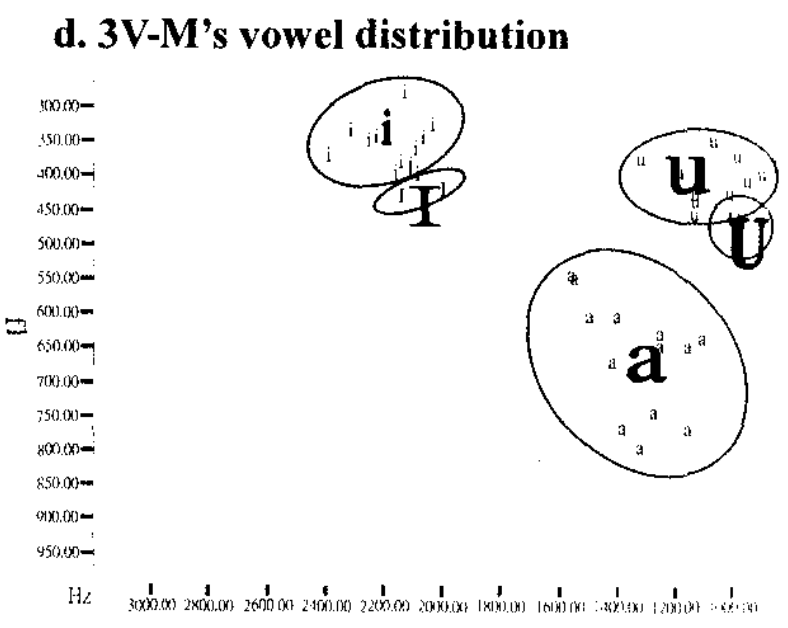

f2 
e. 4V-F's vowel distribution

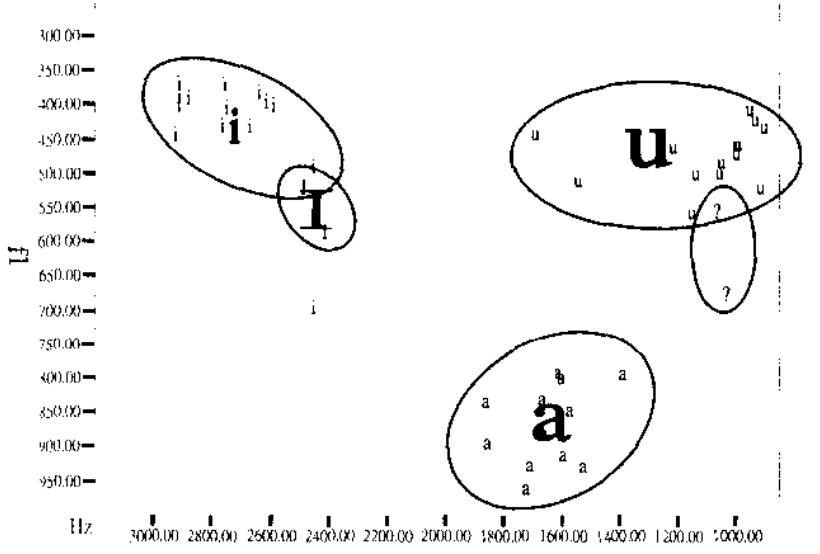

f2

\section{f. 4V-M's vowel distribution}

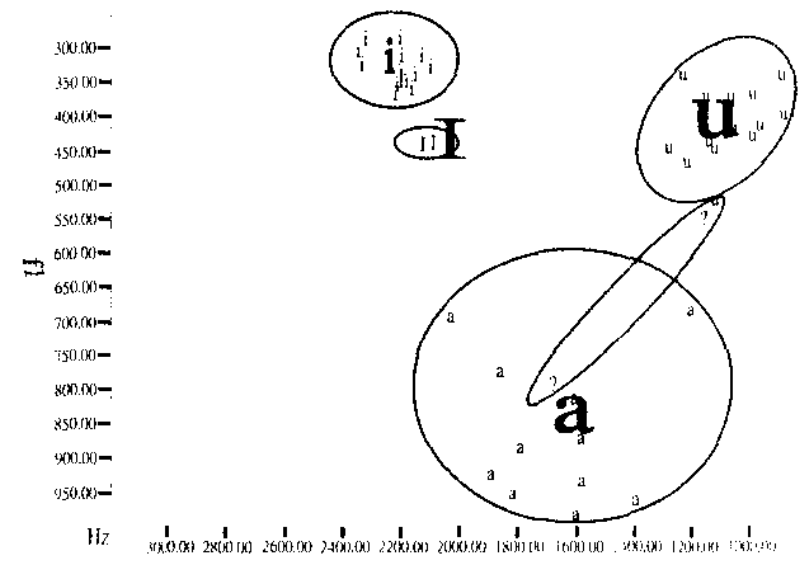

f2

Figure 2. The six speakers' vowel distributions in the unstressed condition.

Vowel distance and vowel space

Figures 3a-f display each speaker's vowel space in both stressed and unstressed conditions. From these displays, we can observe the following: (1) The vowel space seems slightly larger in the stressed than in the unstressed condition, (2) The distance between the vowels [i] and [I/E], or between $[\mathrm{u}]$ and $[\mathrm{U} / \mathrm{O}]$ is greater in the unstressed than in the stressed condition.

a. 5V-F

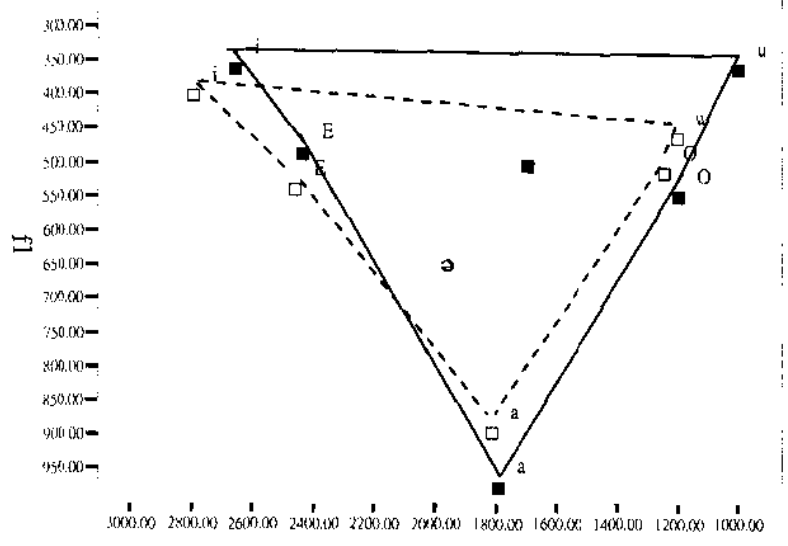

f2 b. 3V-F

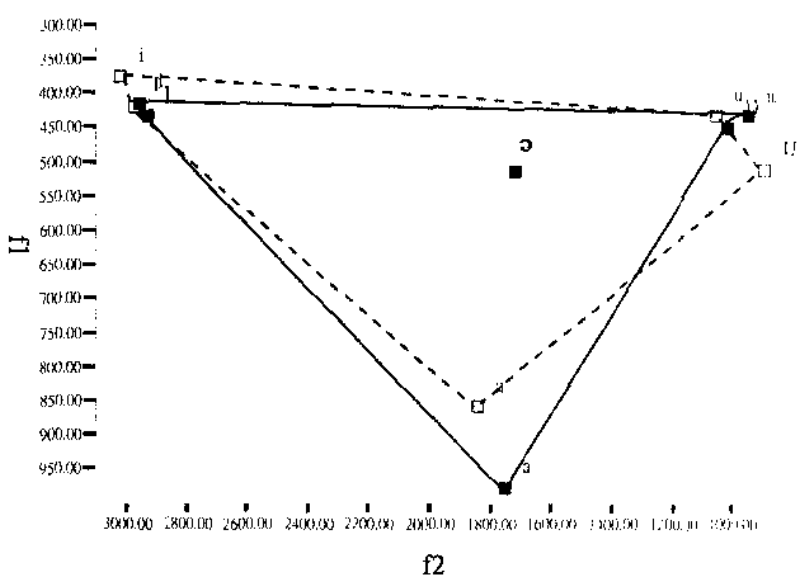


c. $5 \mathrm{~V}-\mathbf{M}$

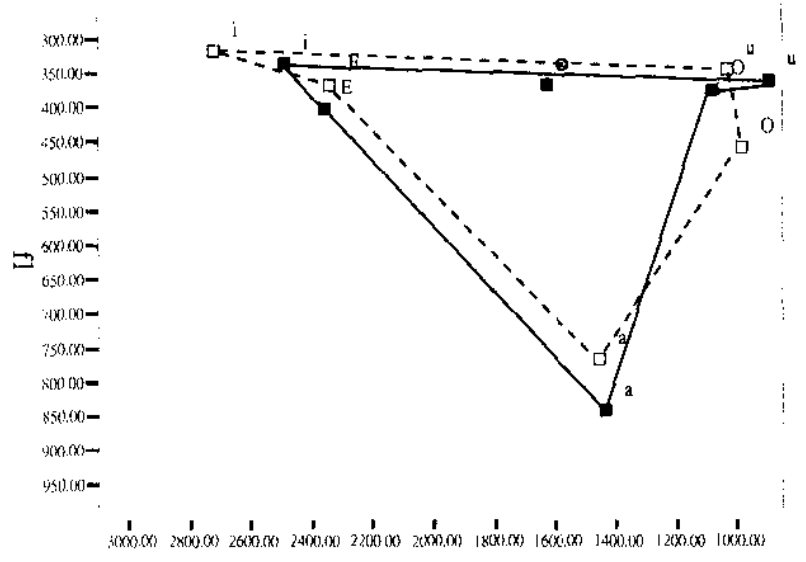

f2

\section{e. $4 \mathrm{~V}-\mathrm{M}$}

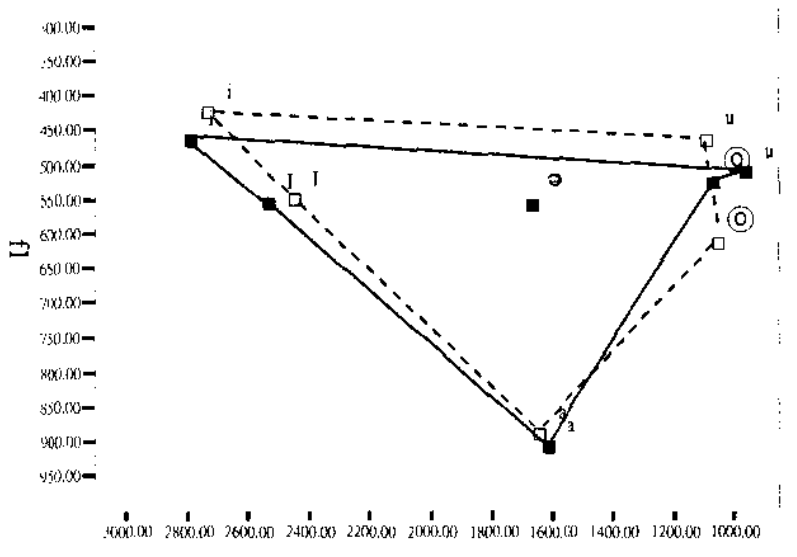

f2

\section{d. 3V-M}

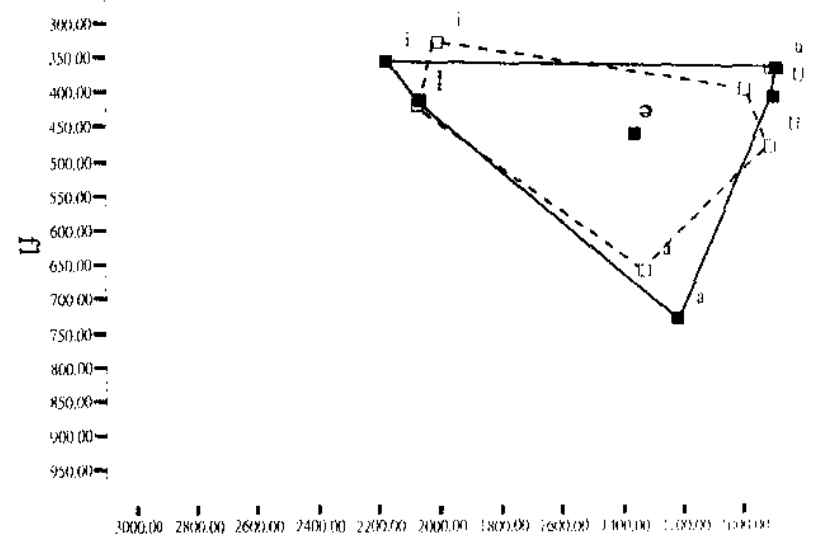

f2

\section{f. 4V-M}

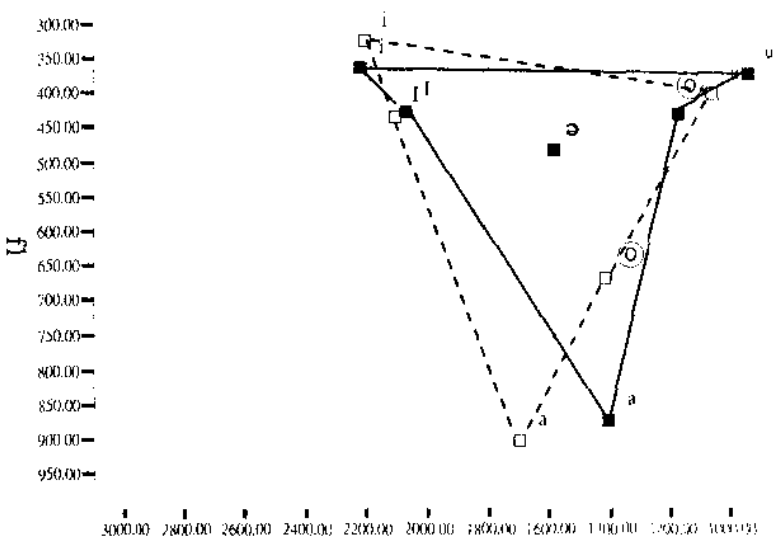

f2

Figure 3. The vowel space of the six speakers. The filled cubes and lines represent the stressed condition, and the hollow cubes and dot lines represent the unstressed condition.

Table 4 and 5 provide further details about the influence of stress on vowel space and vowel distance. The area of the vowel space was calculated by a program ${ }^{7}$ designed to divide the entire space into three triangles; the value of the whole space was derived by adding up the areas of those three triangles. Note that the areas of these spaces (in $\mathrm{Hz}^{2}$ ) do not have any absolute significance, but can be used in a relative sense to compare one vowel space with another. Table 4 shows that the 5-vowel and 3-vowel groups exhibit a shrinking vowel space in the unstressed condition. In contrast, the two speakers in the 4-vowel group exhibit an expanding vowel space in the unstressed condition. 
Table 4. Stress-related variations in vowel space and the stressed/unstressed vowel space ratio. (Unit: $\mathrm{Hz}^{2}$ )

\begin{tabular}{lllll}
\hline & Stressed & Unstressed & Shrinkage & $\begin{array}{l}\text { Ratio } \\
\text { (stressed: unstressed) }\end{array}$ \\
\hline $\mathbf{5 V - F}$ & 506303 & 354415 & Shrink & $\mathbf{1 : 0 . 7 0}$ \\
$\mathbf{3 V - F}$ & 544587 & 451806 & Shrink & $\mathbf{1 : 0 . 8 3}$ \\
$\mathbf{4 V - F}$ & 357832 & 411029 & Expand & $\mathbf{1 : 1 . 1 5}$ \\
\hline $\mathbf{5 V - M}$ & 350324 & 340722 & Shrink & $\mathbf{1 : 0 . 9 7}$ \\
$\mathbf{3 V - M}$ & 244194 & 208259 & Shrink & $\mathbf{1 : 0 . 8 5}$ \\
$\mathbf{4 V - M}$ & 262916 & 304063 & Expand & $\mathbf{1 : 1 . 1 6}$ \\
\hline
\end{tabular}

Table 5 displays the vowel distances between [i] and [I/E], and between [u] and [U/O]. The distances were calculated using the Pythagorean Theorem, and vowel distance was derived by a radical expression.

Table 5. Stress-related variation in vowel distance and stressed/unstressed vowel distance ratio

\begin{tabular}{|c|c|c|c|c|c|c|}
\hline Subject|distance & Stressed & $\begin{array}{l}{[\mathbf{i}]-[\mathbf{I} / \mathbf{E}]} \\
\text { unstressed }\end{array}$ & Ratio & Stressed & $\begin{array}{l}{[\mathbf{u}]-[\mathbf{U} / \mathbf{O}]} \\
\text { unstressed }\end{array}$ & Ratio \\
\hline 5V-F & 252.23 & 358.93 & $1: 1.42$ & 270.77 & 67.29 & $1: 0.25$ \\
\hline $3 V-F$ & 29.21 & 62.68 & 1: 2.15 & 74.58 & 179.30 & 1: 2.40 \\
\hline 4V-F & 270.82 & 306.24 & 1: 1.13 & 110 & 155.88 & $1: 1.42$ \\
\hline$\overline{5 V-M}$ & 146.07 & 381.79 & $1: 2.61$ & 182.26 & 122.16 & $1: 0.67$ \\
\hline 3V-M & 115.63 & 111.26 & 1: 0.96 & 41.95 & 114.32 & $1: 2.73$ \\
\hline 4V-M & 159.61 & 150.44 & 1: 0.94 & 238.26 & 444.13 & 1: 1.86 \\
\hline
\end{tabular}

In general, the distance between $[\mathrm{i}]-[\mathrm{I} / \mathrm{E}]$ and between $[\mathrm{u}]-[\mathrm{U} / \mathrm{O}]$ was observed to increase in the unstressed condition. Thus, the two tables indicate an inverse variation between vowel space and vowel distance -- when vowel space shrinks in the unstressed condition, vowel distance increases.

\section{General discussion}

This section will summarize the three major observations of this study. First, we found the following asymmetry between perception and production: With the exception of the 3-vowel female speaker, even the speakers who did not perceive $[\mathrm{I} / \mathrm{E}]$ to be a distinct vowel in their inventory produced a distinction between [i] and $[\mathrm{I} / \mathrm{E}]$ in terms of $\mathrm{F} 1$ value. Second, stress was found to influence the formant values that constitute the $[\mathrm{u}]-[\mathrm{U} / \mathrm{O}]$ distinction. Vowel space shrunk in the unstressed condition, while vowel distance expanded. Finally, subject-based comparisons across gender groups showed that vowel inventory variation was realized along different parameters for male and female speakers. Female speakers tended to rely on vowel formant values to differentiate vowels in production, while male speakers relied more on vowel space.

\section{Vowel dispersion}

Despite variation in the number of vowels informants perceived, the 
controversial groups $[\mathrm{I} / \mathrm{E}]$ and $[\mathrm{U} / \mathrm{O}]$ exhibited significant formant differences from their uncontroversial counterparts [i] and [u], particularly with respect to vowel height. Individual comparisons showed that for female speakers, vowel height accounted for most of the variation in the number of vowels perceived in the stressed condition. In contrast, male speakers tended to use the parameter of vowel space to determine the number of vowels perceived. Although Table 5 shows a vowel distance distinction between 5-vowel and 3-vowel male speakers, this distinction is not as great as that between 5-vowel and 3-vowel female speakers. Instead, variation in the number of vowels perceived depends mostly upon the size of that speaker's vowel space. Table 4 shows that 5 -vowel male speaker has larger vowel space than 3-vowel male speaker does, and the size of the 4-vowel male's vowel space is in-between.

We conclude from these observations that vowel dispersion operates on two levels: vowel distance and vowel space. The two levels conflict somewhat with previous accounts of vowel distribution, such as Quantal Theory, since the female speakers' high vowels [i] and [u] do not remain at the same height as vowel inventory size changes. In fact, the height of the high vowels seems to be in direct variation with inventory size. The 3-vowel female speaker lowers her [i] and [u], which contributes to the decrease in contrast between the controversial and uncontroversial high vowels. Our observations on vowel distance distinction by female speakers also violate a basic assumption of Adaptive Dispersion -- the acoustic vowel space does not expand as the number of vowels increases. This suggests that maximal perceptual contrast may not necessarily be accomplished by expanding vowel space and vowel distance simultaneously. Together, the two levels provide a strategy for achieving maximal contrast; but in this case, one level seems to provide sufficient contrast to identify vowel inventory members.

\section{Influence of stress on vowel contrastiveness}

It was observed most speakers' vowel spaces shrink when moving from the stressed to unstressed condition, whereas their vowel distances expand. This may be attributed to the insufficient sample size of items containing the controversial vowels $[\mathrm{I} / \mathrm{E}]$ and $[\mathrm{U} / \mathrm{O}]$ in unstressed positions. More extensive data collection is necessary to conduct further research the influence of stress on vowel distance.

In addition, stress had no significant effect on vowel duration. It seems that the influence of stress is confined to shrinkage of the vowel space. A possible phonotactic explanation might involve absence of the schwa in last unstressed syllable position. The lack of the schwa obviates the need to form a contrast with [ə], so according to the principle of minimizing articulatory effort, the vowel space would decrease.

\section{Conclusion}

The findings presented in this paper lead us to propose that the goal of achieving maximal perceptual contrast can be reached in either of two ways: by expanding the vowel space or by increasing the vowel distance. These two levels do not necessarily have to operate in conjunction. The Truku data illustrate both the interaction and the independence of these two levels. Further research is needed, both in terms of more extensive data collection in Truku, 
and in the investigation of other Formosan aboriginal languages, to provide support for these initial findings.

\section{Endnotes}

1. The vowel inventory discussed here does not include the schwa.

2. $\mathrm{Li}$ chose /e/ to represent the fifth vowel, for the purpose of creating symmetry in the vowel inventory.

3. Stress in Truku falls on the penultimate syllable. Chang (2000) described

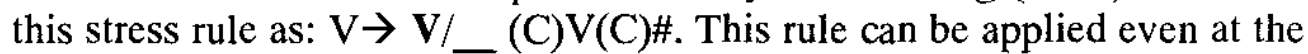
morphological level, since morphological change does not affect the assignment of stress.

4. Yang (1976) and Chang (2000) both indicated a pair of phonological rules in Seediq in which the diphthongs [aw] and [ay] are the ptoto-form of [o] and [e]:

aw $\rightarrow$ o / \# \# ay $\rightarrow \mathrm{e} / \ldots$

Our observation showed that Truku preserved these two diphthongs in a consistent way compared with Seediq. This may account for the sparse occurrence of unstressed [e] and [o] word-finally in our wordlist:

(i) sinaw "wine" (Truku) $>$ sino "wine" (Seediq)

(ii) walay "thread" (Truku) > wale "thread" (Seediq)

5. There is only one sample of the controversial vowel U/O on the 3-vowel female's and 3-vowel male's vowel distributions, because each of them has an outlier which falls outside the range. The 3-vowel female's outlier is 408 $\mathrm{Hz} \mathrm{F} 1$ and $792 \mathrm{~Hz} \quad \mathrm{~F} 2$, while the 3-vowel male's outlier is $500 \mathrm{~Hz} F 1$ and $826 \mathrm{~Hz} \mathrm{~F} 2$. Both are located far back in the vowel space.

6. Due to the non-occurrence of the schwa/o/ in unstressed syllables in Truku, these plots do not show schwa distribution.

7. We would like to thank Cheng Chung-ping, who wrote the vowel space measurement program.

\section{References}

Aboriginal Languages Cultures Education. (2001). Textbook: Truku. Taipei: Council of Indigenous Peoples, Executive Yuan.

Chang, Yung-li. (2000). Seediq Reference Grammar. Taipei: Yuan-Liu.

Flemming, Edward. (1995). Auditory Representations in Phonology. Ph.D. dissertation, UCLA.

Flemming, Edward. (1996). Evidence for constraints on contrast: The dispersion theory of contrast. Chai-Shune K. Hsu (ed.) UCLA Working Papers in Phonology 1, 86-106.

Flemming, Edward. (2001). Contrast and Perceptual Distinctiveness. (ms.)

$\mathrm{Hu}$, Chen-Wei. (2003). Premis on Multimedia Hypertext for Truku Seediq. M.A. thesis, Graduate Institute of Linguistics, Fu Jen Catholic University.

Hung, Ying-sheng. (1993). Taiwan early inhabitants' footstep: the culture \& legend about ten minority tribes. Taipei: China Times Publishing Co.

Huang, Chang-hsing. (2000). Dong sai de ke qun de shou lie wen hua (The culture of hunting in Eastern Seediq). IOE Edited Compilations. 15: 
1-104. Taipei: Institute of Ethnology, Academia Sinica.

Li, Paul Jen-kuei. (1992). Orthographic Systems for Formosan Languages. Taipei: Ministry of Education, Republic of China.

Li, Yi-Yuan et al. (1963). The Atayal of Nan-ao : an ethnological investigation. Institute of Ethnology, Academia Sinica, Monograph: 5-6. Taipei: Institute of Ethnology, Academia Sinica.

Liljencrants, Johan and Lindblom, Björn. (1972). Numerical simulation of vowel quality systems: the role of perceptual contrast. Language. 48(4): 839-862.

Lindblom, Björn. (1986). Phonetic universals in vowel systems. J.J. Ohala and J.J. Jaeger (eds.) Experimental Phonology. Academic Press.

Lindblom, Björn. (1990). Explaining phonetic variation: a sketch of the $\mathrm{H} \& \mathrm{H}$ theory. In W.J. Hardcastle and A. Marchal (eds), Speech Production and Speech Modeling. Dordrecht: Kluwer. 403-39.

Yang, Hsiu-fang. (1976). Phonological Structure of Paran: A Dialect of Sediq. Bulletin of the Institute of History and Philology Academia Sinica. 47: 611-706.

Wen-yu Chiang

Graduate Institute of Linguistics, National Taiwan University

No.1, Sec. 4, Roosevelt Road, Taipei, 106

Taiwan

wychiang@ntu.edu.tw

Fang-mei Chiang

Graduate Institute of Linguistics, National Taiwan University

No.1, Sec. 4, Roosevelt Road, Taipei, 106

Taiwan

r90142010@ntu.edu.tw 